\title{
Anopheline and culicine mosquitoes are not repelled by surfaces treated with the entomopathogenic fungi Metarhizium anisopliae and Beauveria bassiana
}

Ladslaus L Mnyone ${ }^{1,2,3^{*}}$, Constantianus JM Koenraadt ${ }^{2}$, Issa N Lyimo ${ }^{1,4}$, Monica W Mpingwa', Willem Takken², Tanya L Russell ${ }^{1,5,6}$

\begin{abstract}
Background: Entomopathogenic fungi, Metarhizium anisopliae and Beauveria bassiana, are promising bio-pesticides for application against adult malaria mosquito vectors. An understanding of the behavioural responses of mosquitoes towards these fungi is necessary to guide development of fungi beyond the 'proof of concept' stage and to design suitable intervention tools.

Methods: Here we tested whether oil-formulations of the two fungi could be detected and avoided by adult Anopheles gambiae s.s., Anopheles arabiensis and Culex quinquefasciatus. The bioassays used a glass chamber divided into three compartments (each $250 \times 250 \times 250 \mathrm{~mm}$ ): release, middle and stimulus compartments. Netting with or without fungus was fitted in front of the stimulus compartment. Mosquitoes were released and the proportion that entered the stimulus compartment was determined and compared between treatments. Treatments were untreated netting (control 1), netting with mineral oil (control 2) and fungal conidia formulated in mineral oil evaluated at three different dosages $\left(2 \times 10^{10}, 4 \times 10^{10}\right.$ and $8 \times 10^{10}$ conidia $\left.\mathrm{m}^{-2}\right)$.

Results: Neither fungal strain was repellent as the mean proportion of mosquitoes collected in the stimulus compartment did not differ between experiments with surfaces treated with and without fungus regardless of the fungal isolate and mosquito species tested.

Conclusion: Our results indicate that mineral-oil formulations of $M$. anisopliae and $B$. bassiana were not repellent against the mosquito species tested. Therefore, both fungi are suitable candidates for the further development of tools that aim to control host-seeking or resting mosquitoes using entomopathogenic fungi.
\end{abstract}

\section{Introduction}

Laboratory [1-3] and small scale field trials [4,5] have demonstrated that malaria vectors can succumb to entomopathogenic fungus infection. Furthermore, these fungi can equally infect and kill insecticide-resistant and insecticide-susceptible malaria vectors [6-8]. In these views, entomopathogenic fungi are increasingly attracting attention as potential biological control agents against malaria vectors, particularly as they are

\footnotetext{
* Correspondence: llaurent@ihi.or.tz

'Biomedical and Environmental Group, Ifakara Health Institute, P.O. Box 53,

Off Mlabani Passage, Ifakara, Tanzania

Full list of author information is available at the end of the article
}

considered to be evolutionary proof agents, against which resistance is less likely to develop [9]. As such, entomopathogenic fungi have the potential to be used as a chemical insecticide resistance management tool. Fungal infection restored part of the insecticide susceptibility of kdr-resistant anopheline mosquitoes [8] suggesting that fungal infections may extend the lifetime of insecticidal control strategies.

For fungal infection to occur, the conidia need to contact the host, after which they attach to, germinate, and penetrate the cuticle [10]. Once within the host mosquito, the hyphae proliferate whilst exploiting nutritional resources and release toxic metabolites that eventually

C Biomed Central

C 2010 Mnyone et al; licensee BioMed Central Ltd. This is an Open Access article distributed under the terms of the Creative Commons Attribution License (http://creativecommons.org/licenses/by/2.0), which permits unrestricted use, distribution, and reproduction in any medium, provided the original work is properly cited. 
cause sub-lethal and lethal effects to the host [11]. Normally, the host dies from a combination of mechanical damage, nutrient depletion and toxicosis. Mechanical damage causes loss of host's cell membrane integrity and fluids, which in turn leads to decomposition of internal organs [11]. Examples of sub-lethal effects are reduced blood feeding propensity and fecundity of the mosquitoes [12].

Success or failure of infection depends on the nature of host-parasite interaction, which can be altered by physiological, ecological and behavioural conditions of the host [13]. Many studies on host-pathogen interaction in arthropods have focused on physiological and ecological alterations, with little attention paid to behavioural alterations. One of the most important behaviours is the host insect's ability to detect and avoid fungal conidia. Termites [13-15], ants [13] and groundnut beetles [16] were all shown to detect and avoid Metarhizium anisopliae. Behavioural avoidance was also observed in adults of the common flower bug (Anthocoris nemorum) [17] and ladybirds (Coccinella septempunctata) that could both detect and avoid B. bassiana conidia [18]. In the field of mosquito control, if conidia can repel mosquitoes this could minimize mosquito contact with conidia, and thus reduce the efficacy of this control tool. Entomopathogenic fungi that are either non-repellent or attractive would be more desirable unless their ability to repel is strong enough to prevent mosquitoes from entering human houses and biting people. Laboratory studies to optimize fungal formulations of $M$. anisopliae and B. bassiana have been conducted [3] and provide a foundation for conducting field-based efficacy studies. Understanding how mosquitoes respond behaviourally to fungal exposure is thus essential. Avoidance behaviour may hamper the efficacy and the overall epidemiological impact of the fungus. We therefore tested the behavioural response of An. gambiae s.s., An. arabiensis and Culex quinquefasciatus after contacting or detecting conidia of M. anisopliae and B. bassiana. Culex quinquefasciatus are susceptible to entomopathogenic fungi and under field settings they often appear together with malaria vectors, thus both may be targeted. Most importantly, Culex quinquefasciatus cause nuisance and are important vectors of filariasis. Therefore, understanding how they respond to the fungus was also deemed important since targeting both vectors would be more cost-effective and possibly enhance societal adoption of the technology. Behavioural responses can vary with conidia dose [19]; therefore, we tested different conidia doses formulated in pure mineral oil.

\section{Materials and methods Mosquitoes}

Mosquitoes used in this study were obtained from insectary colonies maintained in the Ifakara Health Institute
(IHI), Tanzania. The An. gambiae s.s. colony was established from a population near Njage village, Tanzania, in 1996. The An. arabiensis colony was established from Sagamaganga village, Tanzania in 2007. Larvae and adults were reared using procedures described by Huho et al [20]. The Cx. quinquefasciatus colony was established from Ifakara village, Tanzania in 2009; using similar procedures as with anophelines except that adults were blood-fed on pigeons. The study was performed using 3-7 d old unfed adult female mosquitoes that were starved at least $6 \mathrm{~h}$ before use.

\section{Fungal isolates, formulation and application}

Two fungal isolates were used: 1) M. anisopliae var. anisopliae ICIPE-30, isolated in 1989 from the maize stalk borer, Busseola fusca (Lepidoptera, Noctuidae) in Western Kenya, and 2) B. bassiana I93-825 (IMI 391510), isolated from a chrysomelid beetle (Coleoptera) in the USA. Dry conidia of $M$. anisopliae were produced at IHI, after passaging and re-isolation from infected mosquito cadavers. Conidia were harvested from $15 \mathrm{~d}$ old cultures grown on rice grains. Dry conidia of $B$. bassiana were imported from Penn State University, USA (courtesy M.B. Thomas, Penn State University, USA). Conidia were formulated in highly refined mineral oil, Enerpar (Enerpar M002 ${ }^{\circ}$, BP Southern Africa Ltd). Preparation and application of fungal formulations was done using procedures described by Mnyone et al [3]. After the treatment of exposure netting (conidia formulation or oil) it was left to dry for $24 \mathrm{~h}$ at ambient conditions.

\section{Behaviour chamber}

A glass chamber with three equally sized compartments $(250 \times 250 \times 250 \mathrm{~mm})$ was used: release, middle and stimulus compartments (Figure 1). The release compartment was separated from the middle compartment by a plywood frame fitted with white paper with a square opening at the middle $(50 \times 50 \mathrm{~mm})$ to allow mosquitoes to move into the adjacent compartment. The middle and stimulus compartments were separated by a plywood frame fitted with a piece of polyester netting. The netting contained three rows of circular holes (10 mm diameter) with each row containing three holes. The distance between holes within and between adjacent rows was $50 \mathrm{~mm}$. The ends of the glass chamber were covered with a transparent piece of cloth to prevent mosquitoes from escaping. The different treatments (three concentrations of fungal conidia suspended in mineral oil, mineral oil only and untreated) were applied to the netting separating the middle from stimulus compartments. In each replicate, freshly treated netting was used. To attract mosquitoes into the stimulus compartment, via the exposure netting, a host odour in the form 


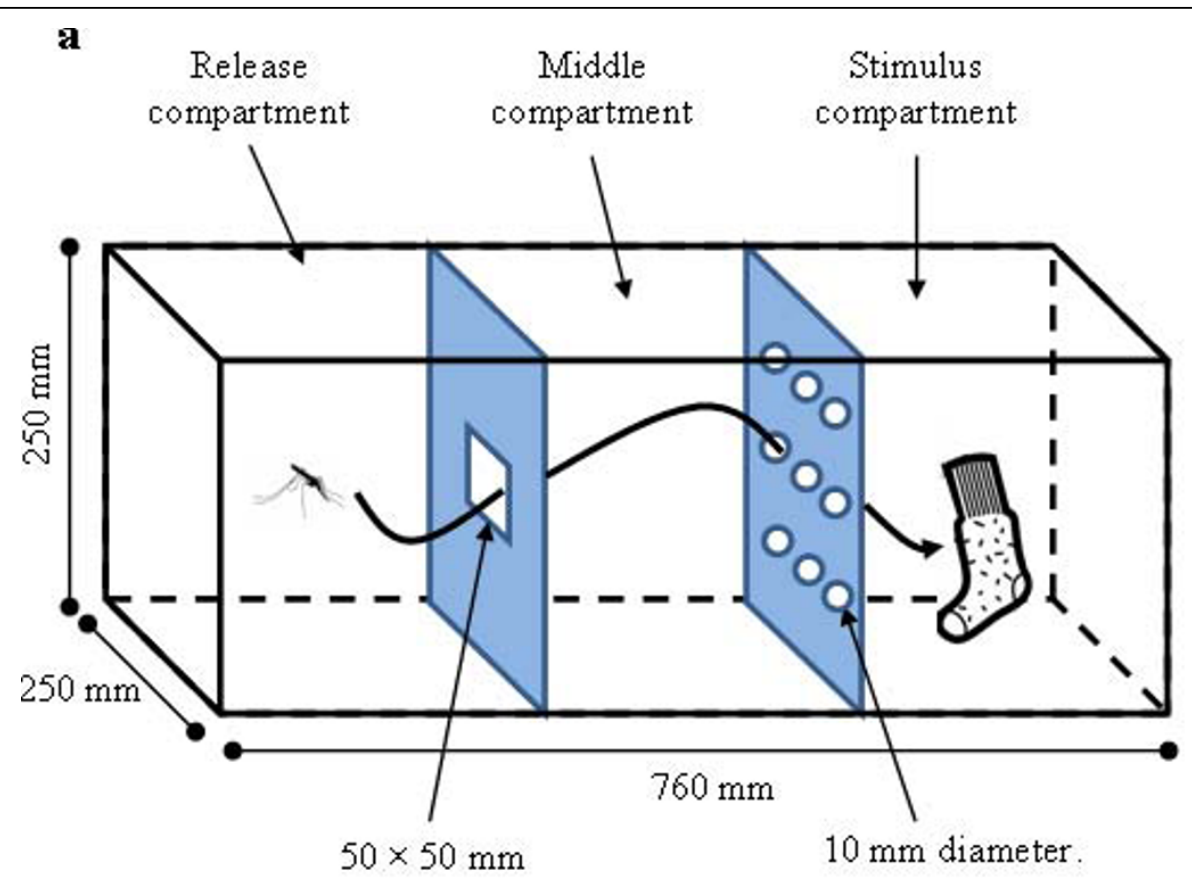

b

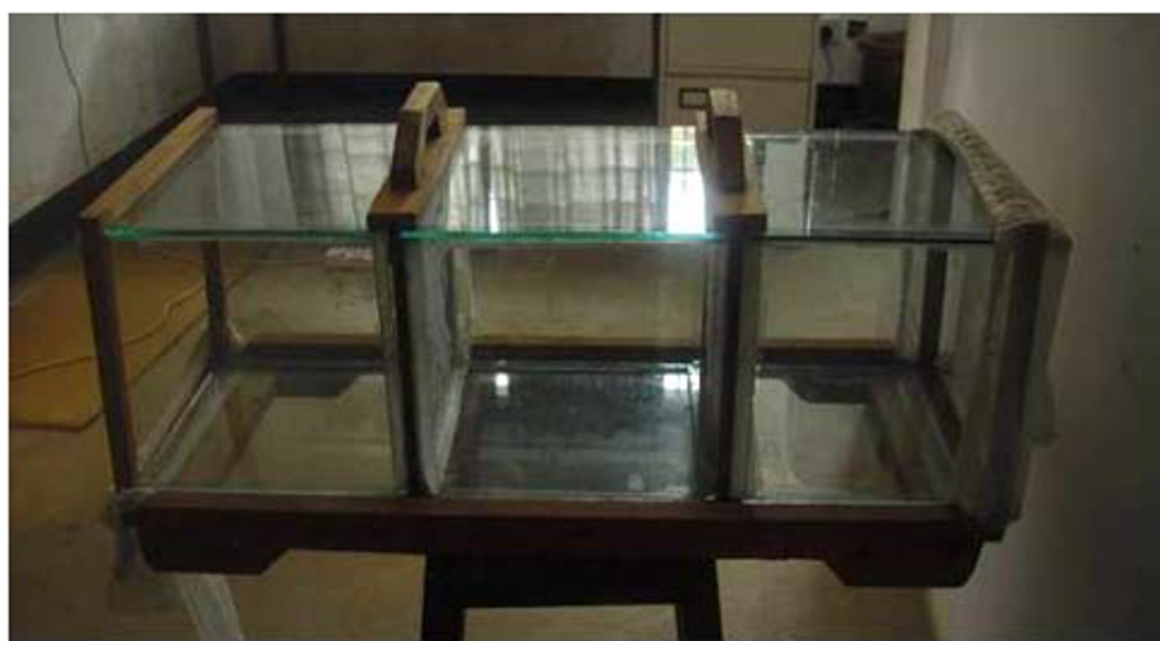

Figure 1 Behavioural chamber with three equally sized compartments: release, middle, and stimulus compartment. A guinea pig (Experiment 1) or a worn sock (Experiment 2) was placed in the stimulus compartment.

of a guinea pig or worn sock was placed into the stimulus compartment. When used, the guinea pig was restrained within a plywood box covered with netting to protect guinea pigs from mosquito bites. The glass chamber was cleaned with distilled water and $70 \%$ alcohol in between trials and left to dry in open air to prevent transferring residual effects to the subsequent trials. Four glass chambers were used in parallel, corresponding to the four different treatments as detailed in the experimental procedures below. Air flow inside the experimental room was passive.

\section{Experimental procedures}

\section{Experiment 1}

Two doses $\left(2 \times 10^{10}\right.$ and $4 \times 10^{10}$ conidia $\left.\mathrm{m}^{-2}\right)$ of $M$. anisopliae and B. bassiana were tested against 
An. gambiae s.s. and An. arabiensis and compared with oil only and untreated controls. The two treatments and controls were run concurrently. Fungal isolates were tested each at a time; and species of mosquitoes were tested against a particular fungal isolate each at a time. About 70 mosquitoes were placed into the release compartment in the early evening $(6: 00 \mathrm{pm})$ and were free to move towards the stimulus compartment overnight. The stimulus compartment for treatments and controls consisted of a guinea pig. The next morning (7:00 am), mosquitoes were collected from each compartment, killed and counted. The trial was repeated four times, each time with fresh mosquitoes, to obtain four replicates for each experimental factor.

\section{Experiment 2}

The set up and procedures were similar as described for Experiment 1, however, with the following exceptions. The experiment tested only B. bassiana against $A n$. gambiae s.s., An. arabiensis as well as Cx. quinquefasciatus. The conidial concentrations tested were: $2 \times 10^{10}$ and $8 \times 10^{10}$ conidia $\mathrm{m}^{-2}$. The stimulus compartment for treatments and controls consisted of socks worn by a human volunteer [21]. One sock was used per each glass chamber. The socks were worn for $12 \mathrm{~h}$; and used immediately after being put off. Each trial was repeated six times to obtain six independent replicates. The treatments and controls were run concurrently.

\section{Data analysis}

The proportion of the mosquitoes released that were collected in the stimulus compartment was the output measure; it was calculated as a ratio of the number in stimulus compartment to the total number of mosquitoes (number in release, middle and stimulus compartments). Data were arcsine transformed to meet the assumption of standard normal distribution; then analysis of variance (ANOVA) was performed to compare different treatments. SPSS version 17 was used.

\section{Results}

\section{Experiment 1}

For M. anisopliae, the mean proportions ( $\pm \mathrm{SE}$ ) of $A n$. gambiae that entered the stimulus (guinea pig) compartment were: untreated control $42.1 \pm 4.2 \%$, mineral oil only $37.9 \pm 5 \%$, conidia dose $2 \times 10^{10} 41 \pm 8.1 \%$ and conidia dose $4 \times 10^{10} 44.9 \pm 5 \%$. This difference was not statistically significant $(\mathrm{F}=0.24 ; \mathrm{df}=3,12 ; p=0.87$; Figure 2). The mean proportions for $A n$. arabiensis were: untreated control $52.8 \pm 4.3 \%$, oil only $49.9 \pm$ $5.2 \%$, conidia dose $2 \times 10^{10} 41.6 \pm 5.2 \%$ and conidia dose $4 \times 10^{10} 49.6 \pm 4.2 \%$. This difference was also not statistically significant $(\mathrm{F}=1.0$; $\mathrm{df}=3,12 ; p=0.43$, Figure 2). For B. bassiana, mean proportions of $A n$. gambiae that entered the stimulus chamber were: untreated control $36.4 \pm 1.2 \%$, mineral oil only treated netting $40.9 \pm 4.6 \%$, conidia dose $2 \times 10^{10} 47.5 \pm 2.8 \%$ and conidia dose $4 \times 10^{10} 44.8 \pm 6.4 \%$. This difference was not statistically significant $(\mathrm{F}=1.27 ; \mathrm{df}=3,12 ; p=$ 0.33; Figure 2). Mean proportions of $A n$. arabiensis were: untreated control $43.48 \pm 4.2 \%$, oil only $45.7 \pm$ $5.6 \%$, conidia dose $2 \times 10^{10} 49.7 \pm 4.4 \%$, and conidia dose $4 \times 10^{10} 47.1 \pm 5.1 \%$. This difference was also not statistically significant $(\mathrm{F}=0.27 ; \mathrm{df}=3,12 ; p=0.84$, Figure 2).

\section{Experiment 2}

Mean proportions of $A n$. gambiae that entered the stimulus (worn sock) compartment were: control $35.4 \pm 2.1 \%$, oil-only control $30.7 \pm 2 \%$, conidia dose $2 \times 10^{10} 30.5 \pm$ $1 \%$ and conidia dose $8 \times 10^{10} 32.7 \pm 2.7 \%$. This difference was not statistically significant $(\mathrm{F}=1.19$; $\mathrm{df}=3,20$; $p=0.34$; Figure 3). Mean proportions for An. arabiensis were: untreated control $32.1 \pm 2.1 \%$, oil only $30.1 \pm 2 \%$, conidial dose $2 \times 10^{10} 31.5 \pm 2.3 \%$ and conidial dose $8 \times 10^{10} 30.1 \pm 2.3 \%$. This difference was not significant $(\mathrm{F}=0.21 ; \mathrm{df}=3,20 ; p=0.89)$. Mean proportions for $C x$. quinquefasciatus were: untreated control $41.5 \pm 1.1 \%$, oil only $39.2 \pm 2.4 \%$, conidia dose $2 \times 10^{10} 36.1 \pm 1.7 \%$ and conidia dose $8 \times 10^{10} 36.4 \pm 3.2 \%$. The difference was also not statistically significant $(\mathrm{F}=1.37$; $\mathrm{df}=3,20$; $p=0.28$ : Figure 3).

\section{Discussion}

Successful fungal infection depends on the host contacting treated surface and receiving a threshold dose of infective conidia [22,23]. Results of our two experimental bioassays indicated no repellency of conidia against the three mosquito species tested: Similar proportions of mosquitoes traversed the netting with and without fungus into the stimulus compartment. Scholte et al [24] observed a moderate repelling effect of $M$. anisopliae dry conidia on An. gambiae s.s. The repelling effect, however, disappeared after the conidia were suspended in vegetable oil. In our study, although a different type of oil was used (mineral oil, Enerpar), the oil might have similarly suppressed the moderate repelling effect of the conidia. In a field study in Tanzania, a large proportion of wild anophelines was found sitting on fungus-impregnated sheet [4]. Possibly, the oil film prevents conidia from free dispersion in the air and thus reduces the probability of flying mosquitoes encountering conidia [24] or masks the conidia odour. Interestingly, there are several other benefits gained from formulating conidia in oils. Conidia are more efficacious when formulated in oil than water [25]. Compared to water, oil as carrier offers better adhesion and spreading of the formulation on the lipophilic insect 
Metarhizium anisopliae ICIPE-30

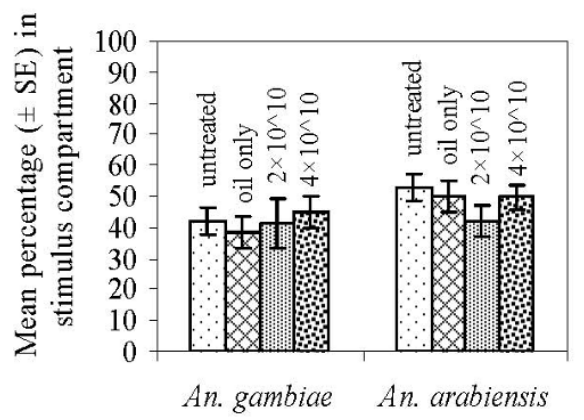

Beauveria bassiana $193-825$

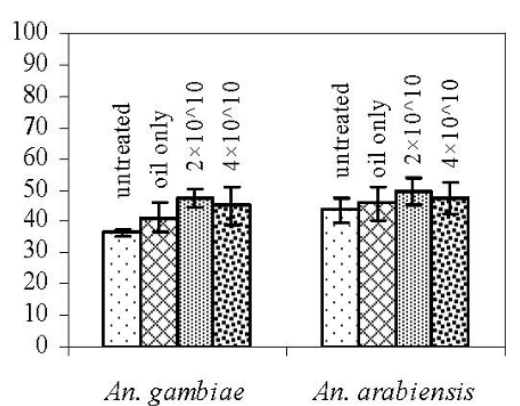

Figure 2 Proportions (Mean \pm SE) of Anopheles gambiae s.s. and Anopheles arabiensis mosquitoes collected in the stimulus compartment with untreated control, mineral oil only control, and two formulations of Metarhizium anisopliae ICIPE-30 and Beauveria bassiana 193-825.

cuticle. Furthermore, the oil can form a film on the host cuticle that acts as a humectant, creating good conditions for conidia to germinate and invade the host [25]. Mineral oil can also improve the tolerance of conidia to extreme temperatures.

The behavioural responses of the arthropod hosts to the fungus may vary with the species of fungus, its virulence and conidia concentration. For example, termites, Coptotermes formosanus, are able to discriminate the species of fungi by their species-specific odors [19]. It was also found that the antennal response increased with increasing concentrations of suspension in the range from $10^{3}$ to $10^{7}$ conidia $\mathrm{ml}^{-1}$ [19]. As such, species-specific evaluations will need to be undertaken before other fungal species or concentrations can be developed for use against specific disease vectors. Importantly, in the present study none of the two fungal isolates were repellent at three conidia doses tested, which represent dose rates that have been recommended for field use [3].
The absence of a repellent effect of $M$. anisopliae and B. bassiana conidia in our experiments could be beneficial in different ways. There is the possibility for infecting mosquitoes by the lure-and-kill principle [26], using for example odour-baited extra-domiciliary targets [5], since the fungal formulations do not have a repellent affect that would interfere with the attraction to lures. Such lack of a repellency of entomopathogenic fungi against target mosquitoes will also enable entomopathogenic fungi to be integrated into use alongside the existing control tools. In a combination strategy with insecticide-treated bed nets (ITNs), mosquitoes deflected due to moderate repellency of synthetic insecticides, could be pushed to alternative surfaces treated with entomopathogenic fungus. In this way, the combined impact of ITNs and entomopathogenic fungi could be synergistic. Theoretical models suggest that when ITNs and fungi are combined the impact on malaria transmission is equivalent to the additive effect of each intervention alone [27].

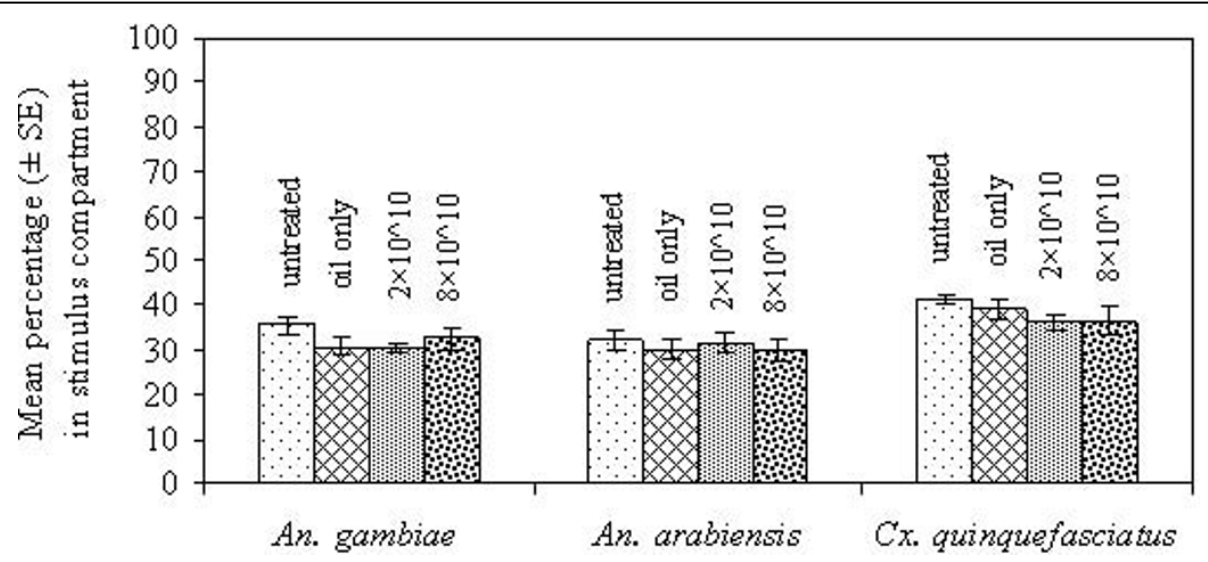

Figure 3 Proportion (Mean \pm SE) of Anopheles gambiae s.s., Anopheles arabiensis and Culex quinquefasciatus collected in the stimulus compartment with untreated control, mineral oil only control, and two formulations of Beauveria bassiana 193-825. 


\section{Conclusion}

Oil-formulations of $M$. anisopliae ICIPE-30 and B. bassiana 193-825 were not found to repel Anopheles gambiae s.s., An. arabiensis and Culex quinquefasciatus, thus, emphasizing the potential of using either fungi for the control of vector mosquitoes.

\section{Acknowledgements}

We wish to thank Paulina Kasanga, Ally Daraja, Kassian Mbina and Emanuel Simfukwe for rearing mosquitoes and technical assistance. We also thank Seth Irish for establishing the colony of Culex quinquefasciatus; and Plasdusi Bakatu for taking care of guinea pigs. This study was funded by Addessium foundation, Reeuwijk, The Netherlands. We thank Frank van Breukelen (Wageningen University), Nina Jenkins and Mathew Thomas (CSIRO/Penn State University) for supplying the M. anisopliae and B. bassiana dry conidia.

\section{Author details}

${ }^{1}$ Biomedical and Environmental Group, Ifakara Health Institute, P.O. Box 53, Off Mlabani Passage, Ifakara, Tanzania. ' Laboratory of Entomology, Wageningen University and Research Centre, P.O. Box 8031, 6700 EH, Wageningen, The Netherlands. ${ }^{3}$ Pest Management Centre, Sokoine University of Agriculture, P.O. Box 3110, Morogoro, Tanzania. ${ }^{4}$ Faculty of Biomedical and Life Sciences, University of Glasgow, 120 University Place, G12 8TA Glasgow, UK. ${ }^{5}$ Vector Group, Liverpool School of Tropical Medicine, Liverpool, L3 5QA, UK. ${ }^{6}$ The University of Queensland, School of Population Health, Australian Centre for Tropical and International Health, Brisbane, 4006, Australia.

\section{Authors' contributions}

Conceived and designed the experiments: LLM, TLR, WT. Performed the experiments: LLM, MWM, INL. Analyzed the data: LLM, CJMK, INL. Wrote the paper: LLM, TLR. Reviewed the paper: CJMK, WT.

\section{Competing interests}

The authors declare that they have no competing interests.

Received: 2 July 2010 Accepted: 27 August 2010

Published: 27 August 2010

\section{References}

1. Blanford S, Chan BHK, Jenkins N, Sim D, Turner RJ, Read AF, Thomas MB: Fungal pathogen reduces potential for malaria transmission. Science 2005, 308:1638-1641

2. Farenhorst M, Farina D, Scholte EJ, Takken W, Hunt RH, Coetzee M, Knols BGJ: African water storage pots for the delivery of the entomopathogenic fungus Metarhizium anisopliae to the malaria vectors Anopheles gambiae s.s. and Anopheles funestus. Am J Trop Med Hyg 2008, 78:910-916.

3. Mnyone LL, Kirby MJ, Lwetoijera DW, Mpingwa MW, Knols BGJ, Takken W, Russell TL: Infection of the malaria mosquito, Anopheles gambiae with two species of entomopathogenic fungi: effects of concentration, coformulation, exposure time and persistence. Malar J 2009, 8:309.

4. Scholte EJ, Ng'habi K, Kihonda J, Takken W, Paaijmans K, Abdulla S, Killeen GF, Knols BGJ: An entomopathogenic fungus for control of adult African malaria mosquitoes. Science 2005, 308:1641-1642.

5. Lwetoijera DW, Sumaye RD, Madumla EP, Kavishe DR, Mnyone LL, Russell TL, Okumu FO: An extra-domiciliary method for delivering entomopathogenic fungi, Metarhizium anisopliae IP 46 against malaria vectors, Anopheles arabiensis. Parasites \& Vectors 2010, 3:18.

6. Farenhorst M, Mouatcho JC, Kikankie CK, Brooke BD, Hunt RH, Thomas MB, Koekemoer LL, Knols BGJ, Coetzee M: Fungal infection counters insecticide resistance in African malaria mosquitoes. Proc Natl Acad Sci USA 2009, 106:17443-17447.

7. Kikankie CK, Brooke BD, Knols BGJ, Koekemoer LL, Farenhorst M, Hunt RH, Thomas MB, Coetzee M: The infectivity of the entomopathogenic fungus Beauveria bassiana to insecticide-resistant and susceptible Anopheles arabiensis mosquitoes at two different temperatures. Malar J 2010, 9:71.
8. Howard AFV, Koenraadt CJM, Farenhorst M, Knols BGJ, Takken W: Pyrethroid resistance in Anopheles gambiae leads to increased susceptibility to entomopathogenic fungi Metarhizium anisopliae and Beauveria bassiana. Malar J 2010, 9:168.

9. Read AF, Lynch PA, Thomas MB: How to make evolution-proof insecticides for malaria control. PLOS Biol 2009, 7:4.

10. Khachatorians GG: Physiology and genetics of entomopathogenic fungi. In Handbook of Applied Mycology, Humans, animals, and insects. Edited by: Arora DK, Ajello L, Mukerji KG. New York: Marcel Dekker Inc; 1991:2.

11. Bush AO, Fernandez JC, Esch GW, Seed JR: Parasitism: The diversity and ecology of animal parasites. Cambridge, UK: Cambridge Univ. Press 2001.

12. Scholte EJ, Knols BG, Takken W: Infection of the malaria mosquito Anopheles gambiae with the entomopathogenic fungus Metarhizium anisopliae reduces blood feeding and fecundity. J Invertebr Pathol 2006, 91:43-49.

13. Roy HE, Steinkraus DC, Eilenberg J, Hajek AE, Pell JK: Bizarre interactions and endgames: entomopathogenic fungi and their arthropod hosts. Ann Rev Entomol 2006, 51:331-357.

14. Yanagawa A, Shimizu S: Resistance of the termite Coptotermes formosanus Shiraki to Metarhizium anisopliae due to grooming. BioControl 2007, 52:72-85.

15. Yanagawa A, Yokohari F, Shimizu S: Defense mechanism of the termite, Coptotermes formosanus Shiraki, to entomopathogenic fungi. J Invertebr Pathol 2008, 97:165-170.

16. Ekesi $\mathrm{S}$, Egwurube EA, Akpa AD, Onu I: Laboratory evaluation of the entomopathogenic fungus, Metarhizium anisopliae for the control of the groundnut bruchid, Caryedon serratus on groundnut. J Stored Prod Res 2001, 37:313-321.

17. Meyling NV, Pell JK: Detection and avoidance of an entomopathogenic fungus by a generalist insect predator. Ecol Entomol 2006, 31:162-171.

18. Omond $\mathrm{E}$ : The overwintering interactions of the seven spot ladybird (Coccinella septempunctata) and the entomopathogenic fungus Beauveria bassiana. PhD thesis Anglia Ruskin University, Cambridge 2007.

19. Yanagawa A, Yokohani F, Shimizu S: The role of antennae in removing entomopathogenic fungi from cuticle of the termite, Coptotermes formosanus. J Insect Sci 2009, 9:9pp.

20. Huho BJ, Ng'habi KR, Killeen GF, Nkwengulila G, Knols BGJ, Ferguson HM: Nature beats nurture: a cast study of the physiological fitness of freeliving and laboratory-reared male Anopheles gambiae s.l. J Exp Biol 2007, 210:2939-2947.

21. Pates HV, Takken W, Stuke K, Curtis CF: Differential behaviour of Anopheles gambiae sensu stricto (Diptera: Culicidae) to human and cow odours in the laboratory. Bull Entomol Res 2001, 91:289-296.

22. Vandenburg JD, Ramos M, Altre JA: Dose -response and age- and temperature-related susceptibility of the diamondback moth (Lepidoptera: Plutellidae) to two isolates of Beauveria bassiana (Hyphomycetes: Moniliaceae). Environ Entomol 1998, 27:1017-1021.

23. Hughes WOH, Petersen KS, Ugelvig LV, Pedersen D, Thomsen L, Poulsen M, Boomsma JJ: Density-dependence and within-host competition in a semelparous parasite of leaf-cutting ants. BMC Evol Biol 2004, 4:45.

24. Scholte EJ, Takken W, Knols BGJ: Pathogenicity of six East African entomopathogenic fungi to adult Anopheles gambiae s.s. (Diptera: Culicidae) mosquitoes. Proc Exp Appl Entomol NEV, Amsterdam 2003, 14:25-29.

25. Luz C, Batagin I: Potential of oil-based formulations of Beauveria bassiana to control Triatoma infestans. Mycopathologia 2005, 160:51-62.

26. Okumu FO, Madumla EP, John A, Lwetoijera DW, Sumaye RD: Attracting, trapping and killing disease-transmitting mosquitoes using odour-baited stations -The Ifakara Odour-Baited Stations. Parasites \& Vectors 2010, 3:12.

27. Hancock PA: Combining fungal biopesticides and insecticide-treated bednets to enhance malaria control. PLoS Comput biol 2009, 5:e1000525.

doi:10.1186/1756-3305-3-80

Cite this article as: Mnyone et al:: Anopheline and culicine mosquitoes are not repelled by surfaces treated with the entomopathogenic fungi Metarhizium anisopliae and Beauveria bassiana. Parasites \& Vectors 2010 3:80. 